\title{
MOVING MESH PARTIAL DIFFERENTIAL EQUATIONS (MMPDES) BASED ON THE EQUIDISTRIBUTION PRINCIPLE*
}

\author{
WEIZHANG HUANG ${ }^{\dagger}$, YUHE REN ${ }^{\dagger}$, AND ROBERT D. RUSSELL ${ }^{\dagger}$
}

\begin{abstract}
This paper considers several moving mesh partial differential equations that are related to the equidistribution principle. Several of these are new, and some correspond to discrete moving mesh equations that have been used by others. Their stability is analyzed and it is seen that a key term for most of these moving mesh PDEs is a source-like term that measures the level of equidistribution. It is shown that under weak assumptions mesh crossing cannot occur for most of them. Finally, numerical experiments for these various moving mesh PDEs are performed to study their relative properties.
\end{abstract}

Key words. equidistribution, moving mesh PDE, adaption

AMS subject classifications. $65 \mathrm{M} 50,65 \mathrm{~L} 50,65 \mathrm{~N} 50$

1. Introduction. Adaptive mesh methods have been widely used in the last decade for solving differential equations that involve large solution variations, such as shock waves, boundary layers, and contact surfaces (e.g., see [11]). It has been amply demonstrated that significant improvements in accuracy and efficiency can be gained by adapting mesh points so that they are concentrated about areas of large solution variation.

For the numerical solution of time-dependent differential equations, adaptive methods can be roughly divided into two categories, static and dynamic. For static methods, the discrete solution and equation are initially defined on a given mesh. During the calculation, a new mesh that might have a different number of nodes from the old mesh is constructed, based on properties of a certain function that measures the goodness of the approximation. The solution is then interpolated from the old mesh to the new mesh, and a new discrete approximation to the solution is defined on the new mesh. The redistribution of the old nodes, the addition of new nodes, and the interpolation of the dependent variables from the old mesh to the new mesh are done at a fixed time. While static methods are generally robust for problems where regions of rapid variation move with time, the continual readjustment can tend to slow the computation, making these methods inefficient.

In this paper, we consider the alternative dynamic methods (often called moving mesh methods). For this type, a mesh equation that involves node speeds is employed to move a mesh having a fixed number of nodes in such a way that the nodes remain concentrated in regions of rapid variation of the solution. The mesh equation and the original differential equation are often solved simultaneously for the physical solution and the mesh. Unlike static methods, interpolation of dependent variables from the old mesh to the new mesh is unnecessary. Among moving mesh methods, the moving finite element method (MFE) of Miller [18], [19] and the moving finite difference method of Dorfi and Drury [7] have aroused considerable interest. The MFE uses a very natural and elegant formulation to control mesh movement. The solution and mesh are both obtained by a process closely associated with equidistribution of one

* Received by the editors October 8, 1992; accepted for publication March 8, 1993. This work was supported in part by the Natural Science and Engineering Research Council of Canada Grant OGP-0008781.

$\dagger$ Department of Mathematics and Statistics, Simon Fraser University, Burnaby, British Columbia V5A 1S6, Canada. 
error measure: the residual of the original equation written in finite element form. While the MFE has been subject to some criticism because of its complexity and sensitivity with respect to certain user defined input parameters [9], proper choice of these parameters unquestionably leads to an efficient method. The method in [7] is based upon a moving mesh equation obtained directly from an equidistribution principle. It is recommended in [9] for actual applications because of its simplicity and its insensitivity of selecting the parameters.

The equidistribution principle, or EP, first introduced by de Boor [5] for solving boundary value problems for ordinary differential equations, involves selecting mesh points such that some measure of the solution error is equalized over each subinterval. It has turned out to be an excellent principle for formulating moving mesh equations. In fact, a number of moving mesh methods have been developed, and almost all are based at some point on an EP. The MFE can be strongly linked to some EPs when it is applied to parabolic differential equations [12]. Adjerid and Flaherty [1], [2] introduce an error estimate to handle mesh movement based on an EP. In [3], [8], [14], [20], and [21] several other moving mesh methods are developed, based directly on EPs. Nevertheless, the constructions are very different, and in their final forms the moving mesh equations appear to be quite different from each other. It has proven to be surprisingly difficult to derive consistently reliable moving mesh equations. In addition to the capability of concentrating sufficient points about regions of rapid variation of the solution, a satisfactory mesh equation should be simple, easy to program, and reasonably insensitive to the choice of its adjustable parameters. As compared with the problem of discretizing the underlying physical equation, this task is purely artificial. That is, the construction of a moving mesh equation cannot be guided completely by physical arguments and must rely on some numerical principles. Furthermore, because most of them have been developed in a discrete form, comparison and theoretical analysis of the moving mesh methods can be difficult.

To facilitate a better understanding of these methods and to allow for a better comparison of their basic properties, we find it useful here to derive continuous moving mesh equations corresponding to the discrete moving mesh equations. In addition, we derive several new continuous moving mesh equations based directly on EPs. Finally, we perform a theoretical and computational study of these continuous moving mesh equations. Besides investigating the relative advantages of the reliable methods (like [7]) for solving PDEs in one space dimension, our ultimate concern is to develop methods for which extension to higher dimensions is possible.

Henceforth, in contrast with a discrete moving mesh equation, which is an ordinary differential equation system, a continuous moving mesh equation will be referred to as a moving mesh partial differential equation, or MMPDE.

An outline of this paper is as follows. In $\S 2$ several different approaches are used to derive a variety of MMPDEs. The theoretical and computational analysis of these MMPDEs is given in $\S \S 3$ and 4 , respectively. Section 5 contains conclusions and further discussion.

2. Moving mesh PDEs. In this section, three approaches will be described to construct MMPDEs for the node speed. The first two are directly based on EPs. For the second one, which is new, the deviation of the moving mesh from the (exact) equidistribution mesh plays a fundamental role in the construction of the MMPDE. For the third approach, although it is based on so-called attraction and repulsion pseudoforces between nodes, the resulting MMPDEs are also closely related to the EP. The first and third approaches have been used previously by many authors to 
develop moving mesh methods, but the mesh equations have usually been represented in a discrete form. Thus, in addition to reviewing discrete mesh equations, we are also deriving their corresponding MMPDEs.

Since all MMPDEs considered in this section are related to the EP, we first give a detailed description of it. Let $x$ and $\xi$ denote the physical and computational coordinates, respectively, both of which are without loss of generality assumed to be the unit interval $[0,1]$ in real space. A one-to-one coordinate transformation between these domains is denoted by

$$
\begin{aligned}
& x=x(\xi, t), \quad \xi \in[0,1] \\
& \text { with } x(0, t)=0, x(1, t)=1,
\end{aligned}
$$

where $t$ denotes time. Hereafter, we shall employ the following notation:

$$
\begin{aligned}
f_{x} & \equiv \frac{\partial f}{\partial x} \\
& \left.\equiv \frac{\partial f}{\partial x}\right|_{t} \text { fixed, } \\
f_{t} & \equiv \frac{\partial f}{\partial t} \\
& \left.\equiv \frac{\partial f}{\partial t}\right|_{x} \text { fixed, } \\
\frac{\partial f}{\partial \xi} & \left.\equiv \frac{\partial f}{\partial \xi}\right|_{t \text { fixed }}=\left.\frac{\partial f}{\partial x}\right|_{t} \text { fixed }\left.\frac{\partial x}{\partial \xi}\right|_{t \text { fixed, }} \\
\dot{f} & \equiv \frac{d f}{d t} \\
& \left.\equiv \frac{\partial f}{\partial t}\right|_{\xi \text { fixed }}=\left.\left.\frac{\partial f}{\partial x}\right|_{t \text { fixed }} \frac{\partial x}{\partial t}\right|_{\xi \text { fixed }}+\left.\frac{\partial f}{\partial t}\right|_{x \text { fixed }}
\end{aligned}
$$

for an arbitrary function $f=f(x, t)=f(x(\xi, t), t)$. Suppose that a uniform mesh is given on the computational domain by

$$
\xi_{i}=\frac{i}{n}, \quad i=0,1, \ldots, n,
$$

where $n$ is a certain positive integer, and denote the corresponding mesh in $x$ by $\left\{x_{0}, x_{1}, \ldots, x_{n}\right\}$. Values of an arbitary function $f$ on this computational mesh will be denoted by

$$
f_{i}=f\left(\xi_{i}, t\right)
$$

For a chosen monitor function $M(x, t)(>0)$ that provides some measure of the computational error in the solution of the underlying physical PDE, the (onedimensional) EP can be expressed in its integral form [22] as

$$
\int_{0}^{x(\xi, t)} M(\tilde{x}, t) d \tilde{x}=\xi \theta(t),
$$

where

$$
\theta(t)=\int_{0}^{1} M(\tilde{x}, t) d \tilde{x}
$$


Differentiating (5) with respect to $\xi$ once and twice, we obtain two differential forms of the EP,

$$
M(x(\xi, t), t) \frac{\partial}{\partial \xi} x(\xi, t)=\theta(t)
$$

and

$$
\frac{\partial}{\partial \xi}\left\{M(x(\xi, t), t) \frac{\partial}{\partial \xi} x(\xi, t)\right\}=0 .
$$

Since EPs (5), (7), and (8) do not contain the node speed $\dot{x}(\xi, t)$, for reasons which will become more apparent later, they will be called quasi-static EPs (QSEPs).

Now we consider how to construct MMPDE based on these QSEPs.

2.1. MMPDEs constructed by time differentiation of QSEPs. In [8], Flaherty et al. differentiate the integral form (5) of the EP with respect to time to obtain a MMPDE

$$
M(x(\xi, t), t) \dot{x}(\xi, t)+\int_{0}^{x(\xi, t)} \frac{\partial}{\partial t} M(\tilde{x}, t) d \tilde{x}=\xi \dot{\theta}(t) .
$$

Then, (9) is discretized in space on the mesh (3) by finite differences to give an ODE system

$$
M_{i}(t) \dot{x}_{i}(t)-M_{i-1}(t) \dot{x}_{i-1}(t)+\int_{x_{i-1}(t)}^{x_{i}(t)} \frac{\partial}{\partial t} M(\tilde{x}, t) d \tilde{x}=\frac{1}{n} \dot{\theta}(t), \quad i=1,2, \ldots, n .
$$

Anderson [3] and Hindman and Spencer [14] discuss conversion of QSEPs into MMPDEs by differentiation with respect to time (see also [11]). For example, Hindman and Spencer consider an equation

$$
\frac{\partial^{2} x}{\partial \xi^{2}}+\left(\frac{\partial x}{\partial \xi}\right)^{3} P=0
$$

where $P$, called a forcing function, is given by

$$
P=\frac{1}{M} \frac{\partial M}{\partial \xi}\left(\frac{\partial x}{\partial \xi}\right)^{-2}
$$

By differentiating (11) with respect to time, they obtain an MMPDE that is equivalent to (9). This is true because (11) can be obtained by taking the second derivative of QSEP (5) with respect to $\xi$. After transforming the original PDE into the computational coordinate system, a time integration algorithm is applied alternately to the transformed PDE and to the matrix equation for the node speeds. The time integration algorithm used is either an implicit first- or second-order method or the explicit second-order predictor-corrector MacCormack method. There is the tendency for the nodes to drift out of optimal adjustment despite the use of the matrix equation for the node speeds. To compensate for this, the node positions are periodically adjusted using a tridiagonal matrix equation based on the approximation of (11) by centered finite differences.

In [20] and [21], Ren and Russell obtain a conservative form of an MMPDE, which is easily derived by differentiating (7) with respect to time. In particular, we have

$$
\frac{\partial}{\partial \xi}(M \dot{x})+\frac{\partial M}{\partial t} \frac{\partial x}{\partial \xi}=\dot{\theta}(t)
$$


and dividing by $\frac{\partial x}{\partial \xi}$ and using (2) and (7), we obtain

$$
\frac{\partial}{\partial x}(M \dot{x})+\frac{\partial M}{\partial t}=\frac{M \dot{\theta}(t)}{\theta}
$$

which is their MMPDE. By construction, it is evident that the MMPDE (14) (or (13)) is mathematically equivalent to the MMPDE (9). However, since (14) is written in a conservative differential form, the (semi-)discrete forms and the stabilities of these MMPDEs may be different. In fact, numerical experiments verify this (see [21]).

The function $\theta(t)$ appearing in (9), (13), and (14) is not convenient for actual computation. We can eliminate $\theta(t)$ by differentiating (9) twice with respect to $\xi$ to obtain

$$
\frac{d}{d t}\left\{\frac{\partial}{\partial \xi}\left(M \frac{\partial x}{\partial \xi}\right)\right\}=0
$$

and

(MMPDE1)

$$
\frac{\partial}{\partial \xi}\left(M \frac{\partial \dot{x}}{\partial \xi}\right)+\frac{\partial}{\partial \xi}\left(\frac{\partial M}{\partial \xi} \dot{x}\right)=-\frac{\partial}{\partial \xi}\left(\frac{\partial M}{\partial t} \frac{\partial x}{\partial \xi}\right)
$$

or by differentiating (13) once to obtain

$$
\frac{\partial^{2}}{\partial \xi^{2}}(M \dot{x})=-\frac{\partial}{\partial \xi}\left(\frac{\partial M}{\partial t} \frac{\partial x}{\partial \xi}\right)
$$

Recall that these MMPDEs are obtained by differentiation of a QSEP with respect to time. The process of differentiation implicitly assumes that $x(\xi, t)$ satisfies this QSEP at any time. This is of course not generally true in actual computations. Moreover, from the boundary conditions on $x(\xi, t)$, these MMPDEs have zero speed solutions if $\frac{\partial M}{\partial t}$ is zero. This means that the mesh does not move if $M(x, t)$ is independent of $t$ regardless of what the initial mesh is. Therefore, $\frac{\partial M}{\partial t}$ might be regarded as the source of mesh movement, so its computation should be important. Unfortunately, in actual applications $\frac{\partial M}{\partial t}$ is often not easy to calculate.

2.2. MMPDEs involving a correction term. From the analysis in $\S 2.1$, we see that it is desirable to derive an MMPDE in such a way that the deviation of the computed mesh from the equidistribution mesh plays a role. Here, the EP (8) will be employed, since $\theta(t)$ does not appear for this QSEP. We require the mesh to satisfy the QSEP at the later time $t+\tau(0<\tau \ll 1)$, instead of at $t$. That is, the mesh satisfies

$$
\frac{\partial}{\partial \xi}\left\{M(x(\xi, t+\tau), t+\tau) \frac{\partial}{\partial \xi} x(\xi, t+\tau)\right\}=0 .
$$

Condition (17) gives a relaxation time $\tau$ for the mesh to satisfy the QSEP. We can also regard (17) as a condition to regularize the mesh movement. Using the expansions

$$
\begin{aligned}
\frac{\partial}{\partial \xi} x(\xi, t+\tau)= & \frac{\partial}{\partial \xi} x(\xi, t)+\tau \frac{\partial}{\partial \xi} \dot{x}(\xi, t)+O\left(\tau^{2}\right) \\
M(x(\xi, t+\tau), t+\tau)= & M(x(\xi, t), t)+\tau \dot{x} \frac{\partial}{\partial x} M(x(\xi, t), t) \\
& +\tau \frac{\partial}{\partial t} M(x(\xi, t), t)+O\left(\tau^{2}\right)
\end{aligned}
$$


in (17) and dropping higher-order terms, we obtain the MMPDE

(MMPDE2)

$$
\frac{\partial}{\partial \xi}\left(M \frac{\partial \dot{x}}{\partial \xi}\right)+\frac{\partial}{\partial \xi}\left(\frac{\partial M}{\partial \xi} \dot{x}\right)=-\frac{\partial}{\partial \xi}\left(\frac{\partial M}{\partial t} \frac{\partial x}{\partial \xi}\right)-\frac{1}{\tau} \frac{\partial}{\partial \xi}\left(M \frac{\partial x}{\partial \xi}\right)
$$

which can be rewritten as

$$
\frac{d}{d t}\left\{\frac{\partial}{\partial \xi}\left(M \frac{\partial x}{\partial \xi}\right)\right\}=-\frac{1}{\tau} \frac{\partial}{\partial \xi}\left(M \frac{\partial x}{\partial \xi}\right)
$$

or

$$
\frac{\partial^{2}}{\partial \xi^{2}}(M \dot{x})=-\frac{\partial}{\partial \xi}\left(\frac{\partial M}{\partial t} \frac{\partial x}{\partial \xi}\right)-\frac{1}{\tau} \frac{\partial}{\partial \xi}\left(M \frac{\partial x}{\partial \xi}\right) .
$$

Compared to (MMPDE1), (MMPDE2) contains the additional "correction" term

$$
-\frac{1}{\tau} \frac{\partial}{\partial \xi}\left(M \frac{\partial x}{\partial \xi}\right)
$$

which measures how closely the mesh $x(\xi, t)$ satisfies the QSEP (see (8)). When $x(\xi, t)$ is not equidistributed, (MMPDE2) moves the mesh toward equidistribution even when $M(x, t)$ is independent of $t$. In this sense, the often difficult to calculate term $\frac{\partial M}{\partial t}$ is less important for (MMPDE2) than for (MMPDE1). Therefore, in principle it may be argued that it is reasonable to drop the term $\frac{\partial x}{\partial \xi} \frac{\partial}{\partial t} M$ or both $\frac{\partial x}{\partial \xi} \frac{\partial}{\partial t} M$ and $\dot{x} \frac{\partial}{\partial \xi} M$ in (MMPDE2). These give the simplifications

(MMPDE3)

$$
\frac{\partial^{2}}{\partial \xi^{2}}(M \dot{x})=-\frac{1}{\tau} \frac{\partial}{\partial \xi}\left(M \frac{\partial x}{\partial \xi}\right)
$$

and

(MMPDE4)

$$
\frac{\partial}{\partial \xi}\left(M \frac{\partial \dot{x}}{\partial \xi}\right)=-\frac{1}{\tau} \frac{\partial}{\partial \xi}\left(M \frac{\partial x}{\partial \xi}\right)
$$

The approach of formulating MMPDEs with a correction term used in this section is quite simple and is based directly on the EP. A desirable feature of this approach is that it can be directly extended to higher space dimensions if a formula for an equidistribution principle is available. Such a formula has recently been derived in [15], and this extension is under investigation.

2.3. MMPDEs based on attraction and repulsion pseudoforces. In this section, we shall review some moving mesh methods that are based on attraction and repulsion pseudoforces between nodes. A node attracts others when a measure of the truncation error at this point is larger than average. If the measure is smaller than average, the neighboring nodes are repelled. We shall also discuss the MMPDEs that correspond to various discrete mesh equations that have been derived by this approach.

Methods considered here compute node speeds in response to deviation in an error measure from some average value. An error measure, denoted by $W$, is generally related to some monitor function. In particular, the error measure is usually expressed by

$$
W_{i}=\int_{x_{i}}^{x_{i+1}} M(\tilde{x}, t) d \tilde{x}
$$


where $M$ is a certain error function. It will be useful to interpret this as a discrete form of

$$
W=M \frac{\partial x}{\partial \xi}
$$

although the function $M$ here may be slightly different from that in (22). This may be motivated, e.g., by taking a simple approximation for (22) like the midpoint rule,

$$
W_{i} \approx M_{i+1 / 2}\left(x_{i+1}-x_{i}\right) .
$$

The error functions are often chosen to be proportional to the first and/or second derivatives of the physical solutions, and probably the most common choices in practice are the arclength and curvature monitor functions.

In [4], Anderson computes the node speed by

(MMPDE5)

$$
\dot{x}=\frac{1}{\tau} \frac{\partial}{\partial \xi}\left(M \frac{\partial x}{\partial \xi}\right)
$$

where $\tau$ is a positive constant. Regarding $W$ as an error indicator, one sees from (24) that (MMPDE5) moves the nodes towards regions where the error is large. It also forces the mesh to have zero speed whenever the mesh is equidistributed.

We now consider the method in [1] and [2]; the general observations will also apply for the similar methods in [17] and [10]. Adjerid and Flaherty use an error estimate $E$ to control mesh movement. The node speed is determined by

$$
\dot{x}_{i+1}-\dot{x}_{i}=-\lambda\left(W_{i}-\bar{W}\right),
$$

where $\lambda$ is a positive parameter, $W_{i}$ is an error indicator on the subinterval $\left(x_{i}, x_{i+1}\right)$, and $\bar{W}$ is the average of the $W_{i}$ values. When $W_{i}$ is chosen as the square of the local-error estimate in $H^{1}, W$ satisfies (23) with

$$
M=(E)^{2}+\left(\frac{\partial E}{\partial x}\right)^{2} .
$$

By eliminating $\bar{W}$ by differencing two consecutive interval equations, (25) reads

$$
\dot{x}_{i+1}-2 \dot{x}_{i}+\dot{x}_{i-1}=-\lambda\left(W_{i}-W_{i-1}\right) .
$$

If we denote $\lambda$ by $\frac{1}{\tau}$ and use (23), we can regard (27) as a centered finite difference approximation of the MMPDE

(MMPDE6)

$$
\frac{\partial^{2} \dot{x}}{\partial \xi^{2}}=-\frac{1}{\tau} \frac{\partial}{\partial \xi}\left(M \frac{\partial x}{\partial \xi}\right)
$$

Indeed, most of the properties of the discrete mesh equation (27) can be derived from (MMPDE6).

In [16], Hyman and Larrouturou obtain two MMPDEs corresponding to what they call an elliptic equation method and linear approximation method. These equations are

$$
\frac{\partial}{\partial x}\left(W \frac{\partial \dot{x}}{\partial x}\right)=-\frac{\beta}{\tau} \frac{\partial W}{\partial x}
$$


and

$$
\frac{\partial^{2}}{\partial x^{2}}(W \dot{x})=-\frac{\beta}{\tau} \frac{\partial W}{\partial x}
$$

where $W$ is a so-called mesh function and $\beta$ (a positive constant) determines the relaxation time with respect to the timescale $\tau$. This timescale is chosen adaptively based on the time variation of the physical solution.

The derivation of Hyman and Larrouturou for these MMPDEs, which is outlined below, is based on the equation

$$
\dot{W}_{i+1 / 2}=\frac{\beta}{\tau}\left(\bar{W}-W_{i+1 / 2}\right),
$$

where $\bar{W}$ is the average of the $W_{i+1 / 2}$ values. They approximate (30) by

$$
\frac{\partial W_{i+1 / 2}}{\partial \Delta x_{i+1 / 2}} \Delta \dot{x}_{i+1 / 2}=\frac{\beta}{\tau}\left(\bar{W}-W_{i+1 / 2}\right),
$$

where $\Delta x_{i+1 / 2}$ denotes $x_{i+1}-x_{i}$. Assuming $W \rightarrow 0$ as $\Delta x \rightarrow 0$ and using the approximation

$$
W_{i+1 / 2}=\Delta x_{i+1 / 2} \frac{\partial W_{i+1 / 2}}{\partial \Delta x_{i+1 / 2}}
$$

we can rewrite $(31)$ as

$$
\frac{\partial \Delta \dot{x}_{i+1 / 2}}{\partial \Delta x_{i+1 / 2}}=\frac{\beta}{\tau} \frac{\bar{W}-W_{i+1 / 2}}{W_{i+1 / 2}} .
$$

Multiplying by $W_{i+1 / 2}$ and taking the difference over two consecutive intervals gives

$$
W_{i+1 / 2} \frac{\partial \Delta \dot{x}_{i+1 / 2}}{\partial \Delta x_{i+1 / 2}}-W_{i-1 / 2} \frac{\partial \Delta \dot{x}_{i-1 / 2}}{\partial \Delta x_{i-1 / 2}}=-\frac{\beta}{\tau}\left(W_{i+1 / 2}-W_{i-1 / 2}\right),
$$

which is just a discrete approximation of the MMPDE (28). Similarly, (29) can be obtained when focusing on a particular mesh function that is sufficiently simple so that $\dot{x}$ can be explicitly extracted from $\dot{W}$ in (30). In [16], the mesh function considered is

$$
W_{i+1 / 2}=\int_{x_{i}}^{x_{i+1}}\left[a_{0}+\sum a_{1}\left|u_{x}\right|+\sum a_{2}\left|u_{x x}\right|\right] d x
$$

where $u$ denotes the physical solution and $a_{0}, a_{1}$, and $a_{2}$ are weighted coefficients. Using (2) and (23), it is not difficult to see that (28) and (29) can be expressed in the computational coordinate system by

$$
\frac{\partial}{\partial \xi}\left(M \frac{\partial \dot{x}}{\partial \xi}\right)=-\frac{\beta}{\tau} \frac{\partial}{\partial \xi}\left(M \frac{\partial x}{\partial \xi}\right)
$$

and

$$
\frac{\partial^{2}}{\partial \xi^{2}}(M \dot{x})+\frac{\partial}{\partial \xi}\left(M \dot{x} \frac{\partial^{2} x}{\partial \xi^{2}} / \frac{\partial x}{\partial \xi}\right)=-\frac{\beta}{\tau} \frac{\partial}{\partial \xi}\left(M \frac{\partial x}{\partial \xi}\right)
$$


TABLE 1

Summary information for the MMPDEs.

\begin{tabular}{|c|c|c|}
\hline MMPDE & Equivalent Form & Related Reference \\
\hline \multirow{4}{*}{1} & MMPDE1 & present \\
\cline { 2 - 3 } & $(9)$ & {$[6],[8]$} \\
\cline { 2 - 3 } & $(14)$ & {$[20],[21]$} \\
\cline { 2 - 3 } & $(13),(15),(16)$ & present \\
\cline { 2 - 3 } & others & {$[3],[14]$} \\
\hline 2 & MMPDE2 & present \\
\cline { 2 - 3 } & $(19),(20)$ & present \\
\hline 3 & MMPDE3 & present \\
\hline 4 & MMPDE4 & present \\
\cline { 2 - 3 } & $(28)(\beta=1)$ & {$[16]$} \\
\hline 5 & MMPDE5 & {$[4]$} \\
\hline 6 & MMPDE6 & {$[1],[2],[10],[17]$} \\
\hline 7 & MMPDE7 & {$[7]$} \\
\hline
\end{tabular}

respectively. With $\beta=1,(36)$ is just (MMPDE4) so with this interpretation (28) is equivalent to (MMPDE4). While (37) is similar to (MMPDE3), it has an extra term, which is sufficiently complicated and so we shall not discuss it further.

We conclude that the MMPDEs obtained by the approach based on attraction and repulsion pseudoforces between nodes are closely related to the EP. This is not surprising since computing node speeds in response to a deviation in an error measure from some average value has nearly the same effect as equidistributing a monitor function. Unfortunately, it is unclear how this approach can be applied in general to higher space dimensions. Even if this could be done from their construction using discrete moving mesh equations (such as (30)), it is unclear how closely the resulting MMPDE would be related to the EP.

In addition to the moving mesh methods considered herein, there are two other popular types of methods, the moving finite element method [18], [19] and the moving finite difference method in [7]. As of yet we have not found an MMPDE related to the discrete mesh equation in MFE. However, ignoring the spatial smoothing, it is easy to show that the MMPDE

$$
\frac{\partial}{\partial \xi}\left(M \frac{\partial \dot{x}}{\partial \xi}\right)-2 \frac{\partial}{\partial \xi}\left(M \frac{\partial x}{\partial \xi}\right) \frac{\partial \dot{x}}{\partial \xi} / \frac{\partial x}{\partial \xi}=-\frac{1}{\tau} \frac{\partial}{\partial \xi}\left(M \frac{\partial x}{\partial \xi}\right)
$$

corresponds to the discrete mesh equation used in the method of Dorfi and Drury [7]. Notice that if $\tilde{\tau}$ can be well defined by $\frac{1}{\tilde{\tau}}=\frac{1}{\tau}-2 \frac{\partial \dot{x}}{\partial \xi} / \frac{\partial x}{\partial \xi}$, then (MMPDE7) has the same form as (MMPDE4) (with $\tau$ replaced by $\tilde{\tau}$ ), although the practical implications of this relationship are not clear.

2.4. Remarks. In the previous subsections, several MMPDEs have been derived. Their mathematically equivalent forms and related references are given in Table 1.

We have seen that the term (21) appears in (MMPDEs 2-7). This term serves as a source of mesh movement and can also be regarded as a mechanism to pull the mesh back toward equidistribution of a monitor function when it drifts away from equidistribution. In this sense, (MMPDEs 2-7) can be considered to be closely related to the QSEP. 
The parameter $\tau$ is introduced in (MMPDEs 2-7). It represents a timescale to move the mesh to be equidistributed. It serves to prevent temporal oscillations and hence produces a smoother mesh trajectory for $x(\xi, t)$. When solving problems with extremely large solution gradients, the numerical monitor values can be very sensitive to small perturbations in the mesh, and the meshes generated via the spatial equidistribution can have oscillations (see $\S 4$ ). This is detrimental for the numerical time integration and causes difficulty in the iterative solution of the nonlinear equations that arise in the implicit time integration with a stiff solver. A nonmoving mesh could in theory occur for sufficiently large $\tau$. However, for small values of $\tau$, (MMPDEs 27) will become dominated by the QSEP term, the resulting ODEs become stiff, and temporal oscillations may arise. However, while the parameter $\tau$ is critical, in our experience the numerical methods are relatively insensitive to the actual choice of $\tau$ in applications.

In contrast to the other MMPDEs, (MMPDE1) has no correction term and hence has no corresponding parameter. Since it can be obtained by taking the limit $\tau \rightarrow+\infty$ in (MMPDE2), (MMPDE1) and its variations can in some sense be said to take an infinite time to equidistribute the mesh.

(MMPDE1) and (MMPDE2) contain the function $\frac{\partial M}{\partial t}$. This makes them more complicated to use in actual applications than other MMPDEs. (MMPDEs 3-7) are quite simple, with the node speed appearing linearly. This is useful for theoretical purposes since we can integrate directly for the node speed and position (i.e., the mesh itself).

\section{Theoretical analysis of MMPDEs.}

3.1. (MMPDE1). Assume that the mesh $x(\xi, t)$ satisfies MMPDE1 exactly and that a small perturbation $\delta x(\xi, 0)$ is introduced. From the boundary conditions for $x(\xi, t)$, the perturbation must satisfy

$$
\delta x(0, t)=0, \quad \delta x(1, t)=0,
$$

and it is also assumed that

$$
|\delta x(\xi, t)|<<1 .
$$

If no additional errors are introduced for $t>0$, then the perturbed mesh $x+\delta x$ satisfies

$$
\frac{d}{d t}\left\{\frac{\partial}{\partial \xi}\left(M(x+\delta x, t) \frac{\partial(x+\delta x)}{\partial \xi}\right)\right\}=0 .
$$

By linearizing (40) and using (15), to first order we obtain the perturbation equation

$$
\frac{d}{d t}\left\{\frac{\partial^{2}}{\partial \xi^{2}}(M \delta x)\right\}=0
$$

Integrating and using the boundary conditions leads to

$$
\delta x(\xi, t)=\frac{M(x(\xi, 0), 0)}{M(x(\xi, t), t)} \delta x(\xi, 0) .
$$

A discrete form of this equation has been derived in [8] and [6]. For most choices of $M(x, t)$, the function

$$
L(t) \equiv \max _{0 \leq \xi \leq 1} \frac{M(x(\xi, 0), 0)}{M(x(\xi, t), t)}
$$


is likely to increase or to be bounded, and hence we cannot expect (MMPDE1) to produce an asymptotically stable mesh. Nevertheless, for fixed $\xi,(M(x(\xi, 0), 0))$ / $(M(x(\xi, t), t))$ can be sufficiently well behaved that $\delta x(\xi, t)$ remains small over moderately large time intervals.

Interestingly, in [6] and [8] Petzold suggests modifying the discrete mesh equation (10) to

$$
\begin{aligned}
& M_{i}(t) \dot{x}_{i}(t)-M_{i-1}(t) \dot{x}_{i-1}(t)+\int_{x_{i-1}(t)}^{x_{i}(t)} \frac{\partial}{\partial t} M(\tilde{x}, t) d \tilde{x}+\lambda \int_{x_{i-1}(t)}^{x_{i}(t)} M(\tilde{x}, t) d \tilde{x} \\
& =\frac{1}{n} \dot{\theta}(t)+\frac{\lambda}{n} \theta(t), \quad i=1,2, \ldots, n,
\end{aligned}
$$

where $\lambda$ is a positive constant. The numerical results in [6] and [8] show that the resulting mesh is globally (asymptotically) stable. Actually, (44) is a discrete approximation of the MMPDE

$$
\frac{\partial}{\partial \xi}(M \dot{x})+\frac{\partial M}{\partial t} \frac{\partial x}{\partial \xi}+\lambda M \frac{\partial x}{\partial \xi}=\dot{\theta}+\lambda \theta
$$

which upon differentiation is mathematically equivalent to (MMPDE2) with $\lambda=\frac{1}{\tau}$. Thus, $\frac{\partial}{\partial \xi}\left(M \frac{\partial x}{\partial \xi}\right)$ has another interpretation, as a stablizing term in (MMPDEs 2-7).

Integrating (16) with respect to $\xi$ and using the boundary conditions for $x(\xi, t)$, we obtain

$$
\begin{aligned}
\frac{\partial \dot{x}}{\partial \xi}= & {\left[-\frac{M_{t}}{M}+\frac{M_{x}}{M^{2}} \int_{0}^{x} M_{t}(\tilde{x}, t) d \tilde{x}-\frac{\xi M_{x}}{M^{2}} \int_{0}^{1} M_{t}(\tilde{x}, t) d \tilde{x}\right] \frac{\partial x}{\partial \xi} } \\
& +\frac{1}{\tau M} \int_{0}^{1} M_{t}(\tilde{x}, t) d \tilde{x},
\end{aligned}
$$

which is easy to solve for $\frac{\partial x}{\partial \xi}$ in terms of $M$ and its derivatives. Assuming that $\frac{\partial}{\partial \xi} x(\xi, 0)>0,(46)$ implies that $\frac{\partial}{\partial \xi} x(\xi, t)>0$ (i.e., the mesh does not cross) for all $t>0$ so long as

$$
\frac{d}{d t} \int_{0}^{1} M(\tilde{x}, t) d \tilde{x} \geq 0
$$

Otherwise, (MMPDE1) may produce crossing meshes, as we verify from the numerical examples in $\S 4$.

It follows easily that if (15) holds, then

$$
\frac{\partial}{\partial \xi}\left(M(x(\xi, t), t) \frac{\partial}{\partial \xi} x(\xi, t)\right)=\frac{\partial}{\partial \xi}\left(M(x(\xi, 0), 0) \frac{\partial}{\partial \xi} x(\xi, 0)\right) .
$$

Hence, the extent to which the mesh generated by (MMPDE1) satisfies the QSEP is completely determined from the initial mesh by this relationship.

3.2. (MMPDE2). The analysis for (MMPDE2) is similar to that of (MMPDE1). From a linear stability analysis, we have

$$
\delta x(\xi, t)=e^{-\frac{t}{\tau}} \frac{M(x(\xi, 0), 0)}{M(x(\xi, t), t)} \delta x(\xi, 0) .
$$


Thus, if $L(t)$ in (43) increases slower than $e^{-\frac{t}{\tau}}$, (MMPDE2) produces an asymptotically stable mesh.

As in the derivation of (46) from (16), we have from (20) that

$$
\begin{aligned}
\frac{\partial \dot{x}}{\partial \xi}= & {\left[-\frac{M_{t}+M / \tau}{M}+\frac{M_{x}}{M^{2}} \int_{0}^{x}\left(M_{t}+M / \tau\right)(\tilde{x}, t) d \tilde{x}\right.} \\
& \left.-\frac{\xi M_{x}}{M^{2}} \int_{0}^{1}\left(M_{t}+M / \tau\right)(\tilde{x}, t) d \tilde{x}\right] \frac{\partial x}{\partial \xi} \\
& +\frac{1}{\tau M}\left[\int_{0}^{1} M_{t}(\tilde{x}, t) d \tilde{x}+\frac{1}{\tau} \int_{0}^{1} M(\tilde{x}, t) d \tilde{x}\right] .
\end{aligned}
$$

Assuming $\frac{\partial}{\partial \xi} x(\xi, 0)>0,(50)$ implies that the mesh crossing cannot occur for all $t>0$ when

$$
\frac{d}{d t} \int_{0}^{1} M(\tilde{x}, t) d \tilde{x}+\frac{1}{\tau} \int_{0}^{1} M(\tilde{x}, t) d \tilde{x} \geq 0
$$

or

$$
\int_{0}^{1} M(\tilde{x}, t) d \tilde{x} \geq e^{-\frac{t}{\tau}} \int_{0}^{1} M(\tilde{x}, 0) d \tilde{x} .
$$

Obviously, the condition (52) is much weaker than (47).

Integrating (19) with respect to time leads to

$$
\frac{\partial}{\partial \xi}\left(M(x(\xi, t), t) \frac{\partial}{\partial \xi} x(\xi, t)\right)=e^{-\frac{t}{\tau}} \frac{\partial}{\partial \xi}\left(M(x(\xi, 0), 0) \frac{\partial}{\partial \xi} x(\xi, 0)\right) .
$$

Thus, for large time (or small $\tau$ ) the mesh produced by (MMPDE2) always tends to equidistribute the mesh. Moreover, (53) gives a good interpretation of the parameter $\tau$.

3.3. Other MMPDEs. It is also straightforward to prove some results about mesh crossing for (MMPDEs 3, 4, and 6). It can easily be shown that

$$
\begin{aligned}
\frac{\partial \dot{x}}{\partial \xi}= & {\left[-\frac{1}{\tau}+\frac{M_{x}}{\tau M^{2}} \int_{0}^{x} M(\tilde{x}, t) d \tilde{x}-\frac{\xi M_{x}}{\tau M^{2}} \int_{0}^{1} M(\tilde{x}, t) d \tilde{x}\right] \frac{\partial x}{\partial \xi} } \\
& +\frac{1}{\tau M} \int_{0}^{1} M(\tilde{x}, t) d \tilde{x}
\end{aligned}
$$

for (MMPDE3),

$$
\frac{\partial \dot{x}}{\partial \xi}=-\frac{1}{\tau} \frac{\partial x}{\partial \xi}+\frac{1}{\tau M \int_{0}^{1} M^{-1}(x(\tilde{\xi}, t), t) d \tilde{\xi}}
$$

for (MMPDE4), and

$$
\frac{\partial \dot{x}}{\partial \xi}=-\frac{M}{\tau} \frac{\partial x}{\partial \xi}+\frac{1}{\tau} \int_{0}^{1} M(\tilde{x}, t) d \tilde{x}
$$

for (MMPDE6). Thus, if (MMPDEs 3, 4, and 6) are solved exactly, they cannot produce mesh crossings.

For (MMPDE6)

$$
\delta x(\xi, t)=e^{-\frac{1}{\tau} \int_{0}^{t} M(x(\xi, \tilde{t}), \tilde{t}) d \tilde{t}} \delta x(\xi, 0) .
$$

Since $M$ is positive for all $t>0$, this implies that the perturbation $\delta x(\xi, t)$ must decrease with time. 
4. Numerical examples for the MMPDEs. In this section, we shall do a computational study of the MMPDEs. In practice, the MMPDEs are of course solved in conjunction with some underlying physical PDE. Nevertheless, to facilitate our study of the relative advantages and disadvantages of the methods we shall assume that the physical solution $u(x, t)$ is given exactly and only solve the MMPDEs.

For simplicity, $M(x, t)$ is taken as the arclength monitor function

$$
M=\sqrt{1+\left(\frac{\partial u}{\partial x}\right)^{2}} .
$$

For the discretization of the MMPDEs, we use finite difference methods in space. The discrete approximations of (MMPDEs 1-7) on the uniform computational mesh (3) are given, respectively, by

(61) $\frac{d}{d t}\left(E_{i}\right)-\left[\frac{\left(M_{t}\right)_{i+1}+\left(M_{t}\right)_{i}}{2\left(\frac{1}{n}\right)^{2}}\left(x_{i+1}-x_{i}\right)-\frac{\left(M_{t}\right)_{i}+\left(M_{t}\right)_{i-1}}{2\left(\frac{1}{n}\right)^{2}}\left(x_{i}-x_{i-1}\right)\right]=-\frac{E_{i}}{\tau}$,

$$
\begin{gathered}
\frac{M_{i+1}+M_{i}}{2\left(\frac{1}{n}\right)^{2}}\left(\dot{x}_{i+1}-\dot{x}_{i}\right)-\frac{M_{i}+M_{i-1}}{2\left(\frac{1}{n}\right)^{2}}\left(\dot{x}_{i}-\dot{x}_{i-1}\right)=-\frac{E_{i}}{\tau} \\
-\dot{x}_{i}=-\frac{E_{i}}{\tau} \\
\frac{1}{\left(\frac{1}{n}\right)^{2}}\left[\dot{x}_{i+1}-2 \dot{x}_{i}+\dot{x}_{i-1}\right]=-\frac{E_{i}}{\tau} \\
\frac{M_{i+1}+M_{i}}{2\left(\frac{1}{n}\right)^{2}}\left(\dot{x}_{i+1}-\dot{x}_{i}\right)-\frac{M_{i}+M_{i-1}}{2\left(\frac{1}{n}\right)^{2}}\left(\dot{x}_{i}-\dot{x}_{i-1}\right)-2 E_{i} \frac{\dot{x}_{i+1}-\dot{x}_{i-1}}{x_{i+1}-x_{i-1}}=-\frac{E_{i}}{\tau}
\end{gathered}
$$

Here, $E_{i}$ is the discrete approximation of $\frac{\partial}{\partial \xi}\left(M \frac{\partial x}{\partial \xi}\right)$ at $\xi=\xi_{i}$ given by

$$
E_{i}=\frac{M_{i+1}+M_{i}}{2\left(\frac{1}{n}\right)^{2}}\left(x_{i+1}-x_{i}\right)-\frac{M_{i}+M_{i-1}}{2\left(\frac{1}{n}\right)^{2}}\left(x_{i}-x_{i-1}\right) \text {. }
$$

The approximation $\frac{d E_{i}}{d t}$ to $\frac{d}{d t}\left\{\frac{\partial}{\partial \xi}\left(M \frac{\partial x}{\partial \xi}\right)\right\}$ at $\xi=\xi_{i}$ is

$$
\begin{aligned}
\frac{d E_{i}}{d t} & =\frac{M_{i+1}+M_{i}}{2\left(\frac{1}{n}\right)^{2}}\left(\dot{x}_{i+1}-\dot{x}_{i}\right)-\frac{M_{i}+M_{i-1}}{2\left(\frac{1}{n}\right)^{2}}\left(\dot{x}_{i}-\dot{x}_{i-1}\right) \\
& +\frac{\left(M_{x}\right)_{i+1} \dot{x}_{i+1}+\left(M_{x}\right)_{i} \dot{x}_{i}}{2\left(\frac{1}{n}\right)^{2}}\left(x_{i+1}-x_{i}\right)-\frac{\left(M_{x}\right)_{i} \dot{x}_{i}+\left(M_{x}\right)_{i-1} \dot{x}_{i-1}}{2\left(\frac{1}{n}\right)^{2}}\left(x_{i}-x_{i-1}\right) \\
& +\frac{\left(M_{t}\right)_{i+1}+\left(M_{t}\right)_{i}}{2\left(\frac{1}{n}\right)^{2}}\left(x_{i+1}-x_{i}\right)-\frac{\left(M_{t}\right)_{i}+\left(M_{t}\right)_{i-1}}{2\left(\frac{1}{n}\right)^{2}}\left(x_{i}-x_{i-1}\right)
\end{aligned}
$$


where $M_{x}$ and $M_{t}$ are calculated analytically. Although in most practical applications, the monitor function is only given at mesh points, making $M_{x}$ and $M_{t}$ difficult to calculate, it is desirable to use the forms (59)-(61) in order to preserve the conservative properties of (MMPDEs 1-3) (also see (15) and (19)). For the boundary conditions, we use

$$
\dot{x}_{0}=0 \quad \text { and } \quad \dot{x}_{n}=0 .
$$

The discrete MMPDEs (59)-(65), associated with the boundary condition (68), are solved using the stiff ODE solver LSODI (see [13]). Default values of parameters of time integration in LSODI are used (i.e., iopt $=0$ ). The method of time integration is chosen as the backward differentiation formulas (BDF) with chord iteration $(m f=$ 25 ), for which an approximate Jacobian is computed by LSODI internally using finite differences. Other required input data are the initial solution and initial mesh, output times, and local time stepping error tolerances rtol and atol. Throughout, we use

$$
r \text { tol }=\text { atol }=10^{-8} \text { and } \quad n=20 .
$$

For the test of the deviation from the equidistribution mesh, we use the indicator function

$$
E(t)=\max _{2 \leq i \leq n-1}\left|E_{i}(t)\right|
$$

We use the notation

$$
\begin{aligned}
G(t) & =\sum_{i=0}^{n-1} \frac{\left(M_{t}\right)_{i+1}+\left(M_{t}\right)_{i}}{2}\left(x_{i+1}-x_{i}\right) \\
& \sim \int_{0}^{1} \frac{\partial}{\partial t} M(\tilde{x}, t) d \tilde{x} \\
S(t) & =\max _{0 \leq i \leq n} \dot{x}_{i}(t) .
\end{aligned}
$$

Two initial meshes will be used,

$$
x_{i}(0)=\frac{i}{n}, \quad i=0, \ldots, n
$$

and

$$
\left\{\begin{array}{l}
x_{i}(0)=x_{i}^{e m}-\delta\left(x_{i}^{e m}-x_{i-1}^{e m}\right), \quad i=1, \ldots, n-1,0 \leq \delta<1 \\
x_{0}(0)=x_{0}^{e m}, \quad x_{n}(0)=x_{n}^{e m}
\end{array}\right.
$$

where $x_{i}^{e m}, i=0, \ldots, n$, is an equidistribution mesh associated with $M(x, 0)$, which is generated by (MMPDE2) with a method described later in this section. The parameter $\delta$ serves to perturb this equidistribution mesh. The meshes (72) and (73) will be abbreviated by UM and EM, respectively. For results reported, nts and jac denote the total number of time steps and total number of Jacobian computations, respectively. All computations are performed on a SPARC $1+$ in double precision.

Example 4.1

$$
u(x, t)=e^{-\pi^{2} t} \sin (\pi x), \quad 0 \leq x \leq 1
$$


(a)

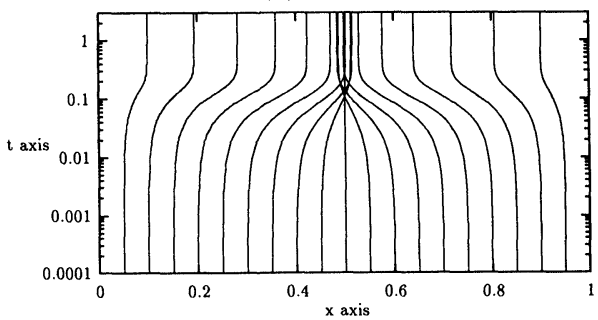

(c)

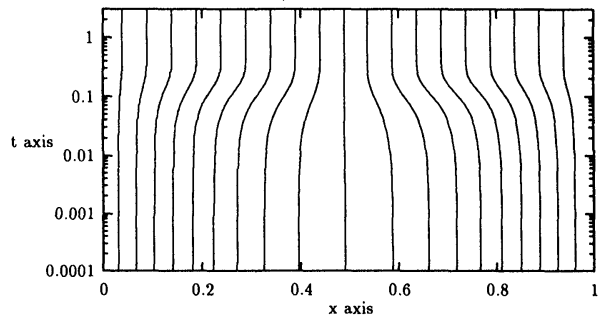

(b)

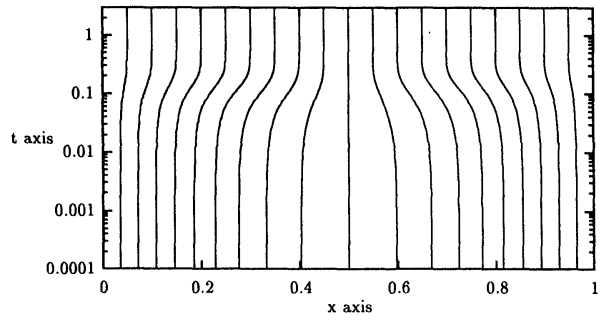

(d)

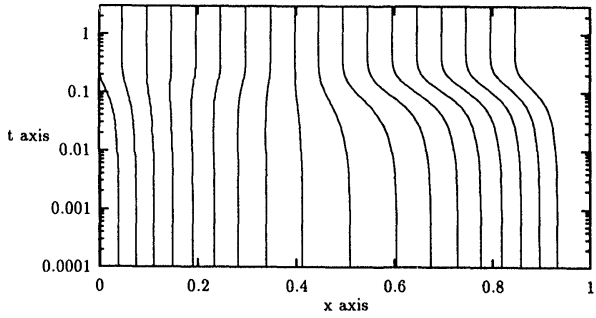

FIG. 1. Example 4.1: Mesh trajectories are generated by (MMPDE1) with initial meshes (a): the UM, (b): the $\operatorname{EM}(\delta=0),(\mathrm{c})$ : the $\operatorname{EM}(\delta=0.1)$ and $(\mathrm{d})$ : the $\operatorname{EM}(\delta=0.9)$.

TABLE 2

Example 4.1. $\tau=10^{-3}$ is used.

\begin{tabular}{|c|cccccc|}
\hline MMPDE & 2 & 3 & 4 & 5 & 6 & 7 \\
\hline nst & 240 & 244 & 241 & 290 & 266 & 231 \\
\hline jac & 34 & 39 & 39 & 45 & 49 & 30 \\
\hline
\end{tabular}

This function is used in [6] to study the stability of some mesh equations. Since $u_{x}(x, t) \rightarrow 0, M(x, t) \rightarrow 1$ as $t \rightarrow+\infty$, and the equidistribution mesh will tend to a uniform mesh in space. The time integration is from $t=0$ to $t=3$.

For (MMPDE1), the mesh trajectories along with the measure of equidistribution level $E(t)$, size of $\frac{\partial M}{\partial t}$ as approximated by $G(t)$ and stability measure $L(t)$ (see $(43)$ ) are plotted in Figs. 1 and 2. Recall from $\S 3.1$ that the deviation from equidistribution depends completely on the initial mesh. Actually, when starting with the initial EM $(\delta=0)$, the computed moving mesh stays almost equidistributed (Fig. 1(b)) with $E(t)$ nearly constant (the bottom curve in Fig. 2(a)). Some oscillations in this curve are caused by the time integration, and they can be reduced using smaller tolerances. However, when the initial UM is used, $E(t)$ is larger (Fig. 2(a)) and the mesh moves in the wrong direction (Fig. 1(a)). Specifically, if the initial UM would adjust to equidistribute $M$, it would move outwards from the center at some time before $t=0.02$ since the node concentration is larger near the middle than it is for the $\operatorname{EM}(\delta=0)$. However, the mesh actually contracts towards the middle until the mesh crossing takes place at about $t=0.1$. Recall from (47) that this is not to be unexpected since $G(t)$ is negative for this example (see Fig. 2(c)).

As shown in Fig. 2(c), $L(t)$ exceeds unity but is bounded. Hence, the mesh might be expected to be stable for small perturbations. To verify this, a computation is performed with a perturbed initial EM $(\delta=0.1)$. The mesh trajectory is plotted in Fig. 1(c). The perturbation is indeed bounded and small. However, if a larger 
(a)

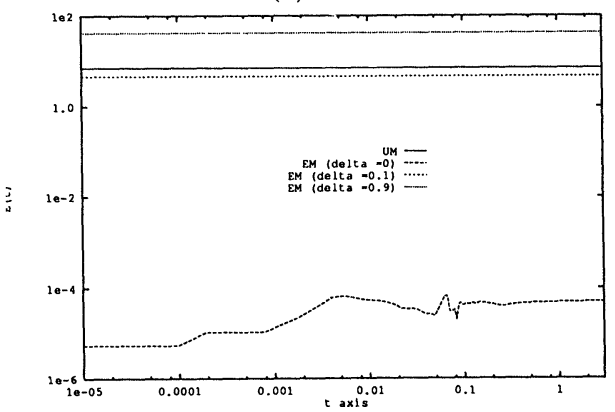

(b)

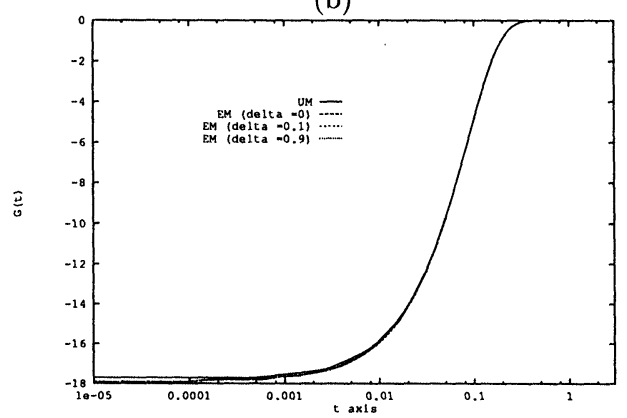

(c) (c)

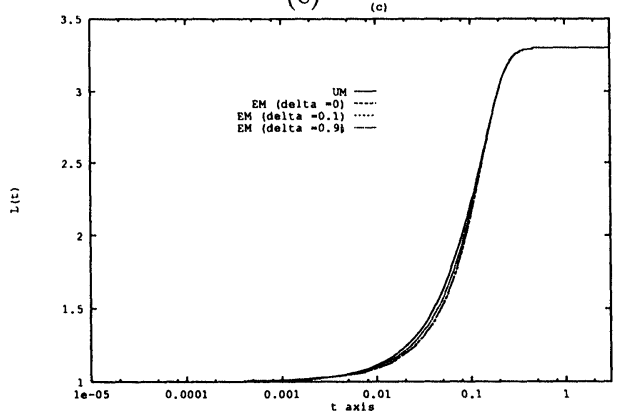

FIG. 2. Example 4.1: The functions $E(t), G(t)$ and $L(t)$ associated with (MMPDE1) are plotted for different initial meshes in (a), (b), and (c), respectively.

(a)

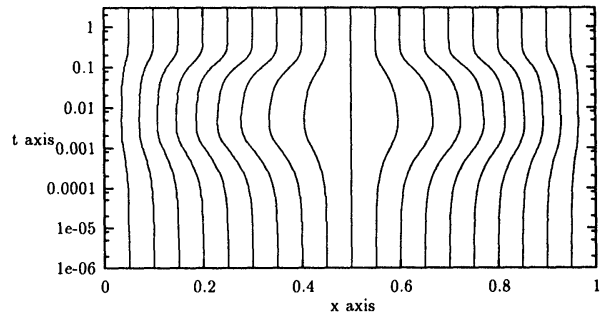

(b)

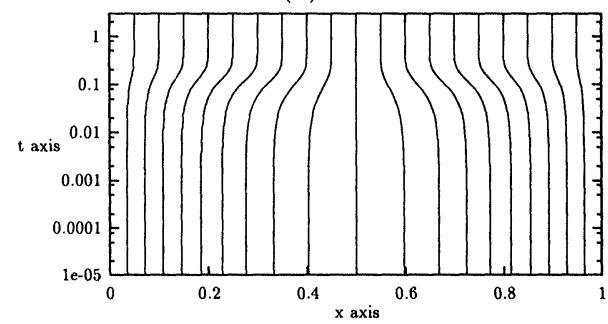

Fig. 3. Example 4.1: Mesh trajectories are generated by (MMPDE4) with initial meshes (a): the UM, (b): the $\operatorname{EM}(\delta=0)$.

perturbation $(\delta=0.9)$ is introduced in the initial mesh, the mesh (Fig. 1(d)) moves far from the equidistribution mesh, and mesh crossing even occurs at the left end.

For (MMPDEs 2-7), the generated meshes are found to be fairly stable, and no mesh crossing occurs. Representative results are illustrated in Fig. 3, which shows the mesh trajectories produced by (MMPDE4) with the initial UM and EM $(\delta=0)$. These two trajectories are nearly the same after about $t=0.01$. In Figs. 4(a) and $4(\mathrm{~b}), E(t)$ and the mesh velocity $S(t)$ are plotted for (MMPDEs 2-7). Figure 4(a) shows that $E(t)$ decreases, so the meshes move toward equidistribution as expected, due to the presence of the correction term in the MMPDE. The timescale of the movement depends on the particular MMPDE and value of $\tau$. From Fig. 4, for the fixed $\tau$ value (MMPDE5) has the shortest timescale, followed by (MMPDE6). 
(a)

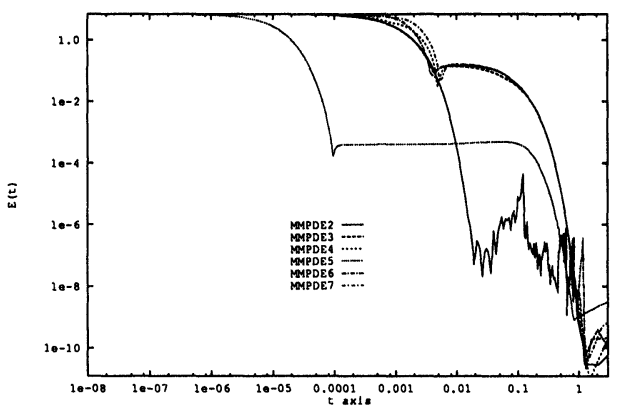

(b)

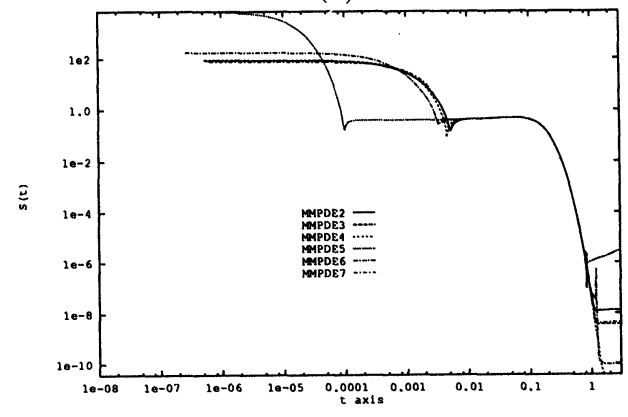

FIG. 4. Example 4.1: The functions $E(t)$ and $S(t)$ for (MMPDEs 2-7) with the initial mesh $\mathrm{UM}$ in (a) and (b), respectively. Here, $\tau=10^{-3}$ is used.

(c)

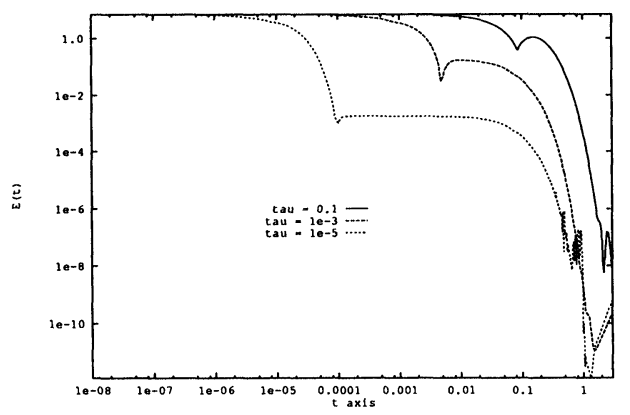

FIG. 5. Example 4.1: The function $E(t)$ is obtained by (MMPDE4) with the initial uniform mesh for different values of $\tau$.

While (MMPDE2) gives an initially monotonically decreasing function $E(t)$, it is also sensitive to the accuracy of the time integration when $E(t)$ is small. Figure 5 shows $E(t)$ for (MMPDE4) with $\tau$ values $0.1,10^{-3}$, and $10^{-5}$. Values of nst and jac for several runs are listed in Table 2.

Example 4.2.

$$
\left\{\begin{array}{l}
u(x, t)=\frac{1}{2}[1-\tanh (c(t)(x-t-0.4))] \\
c(t)=1+\frac{\left(10^{3}-1\right)}{2}[1+\tanh (100(t-0.2))], \quad 0 \leq x \leq 1, \quad 0 \leq t \leq 0.55
\end{array}\right.
$$

This solution represents a wave that moves towards $x=1$. The wave is smooth at the beginning and then suddenly develops a steep gradient at about $t=0.2$.

Computations are performed with an initial uniform mesh, which, for this example, is a good approximation $(E(0) \sim 0.133)$ of the initial equidistribution mesh. Hence, (MMPDE1) could be expected to work well. Indeed, this is the case, as can be seen from the mesh trajectories shown in Fig. 7(a).

The functions $E(t)$ and $S(t)$ for (MMPDEs 1-6) with $\tau=10^{-3}$ are plotted in Figs. 6(a) and 6(b), respectively. Notice that there are significant oscillations for the top three curves in Fig. 6(a), which correspond to (MMPDEs 3, 4, and 6). Actually, similar but smaller oscillations occur for other curves. This can also be readily seen 
(a)

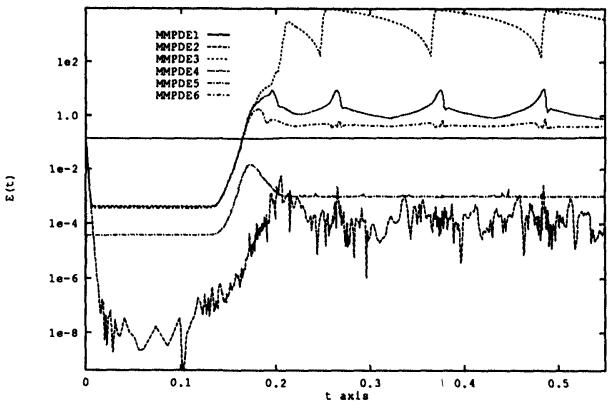

(b)

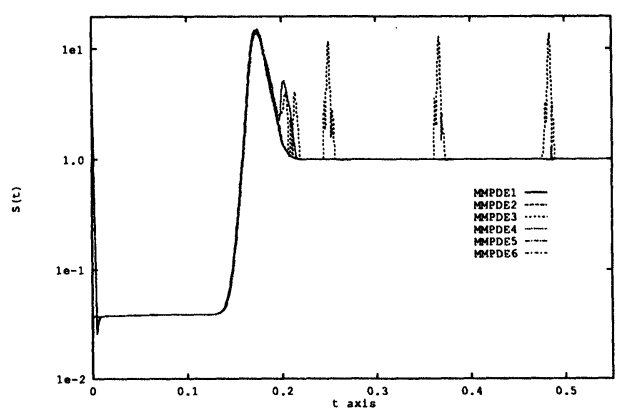

FIG. 6. Example 4.2: The functions $E(t)$ and $S(t)$ for (MMPDEs 1-6) with the initial mesh $\mathrm{UM}$ are plotted in (a) and (b), respectively. Here, $\tau=10^{-3}$ is used.

(a)

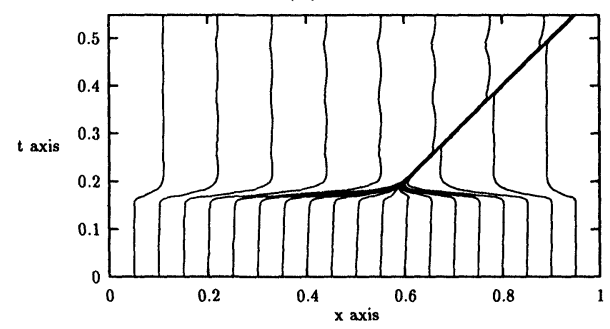

(c)

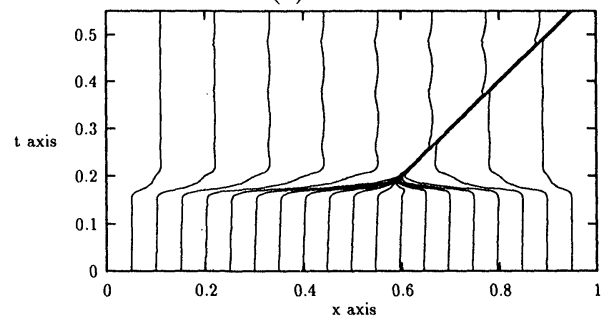

(b)

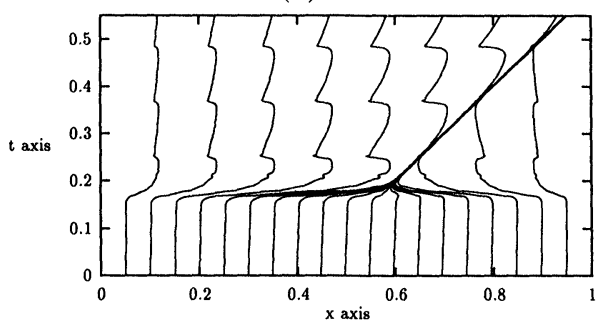

(d)

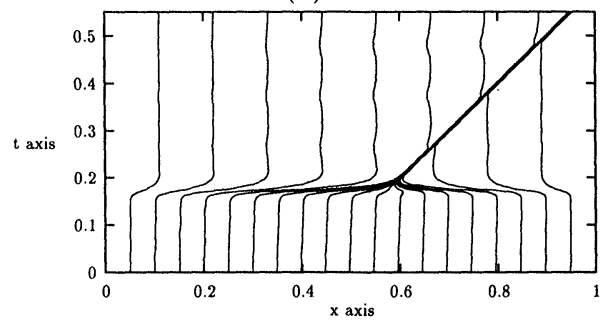

FIG. 7. Example 4.2: Mesh trajectories, which start with the initial uniform mesh, are generated (a) by (MMPDE1), (b) by (MMPDE3) with $\tau=10^{-3}$, (c) by (MMPDE4) with $\tau=10^{-3}$, and (d) by (MMPDE3) with $\tau=10^{-5}$.

for the mesh trajectories shown in Figs. $7(\mathrm{a})-7(\mathrm{c})$. This phenomenon is due to the fact that the mesh is adjusted to equidistribute the monitor function, and some nodes go into and some out of the region of high solution gradient as the wave moves. Since the wave is fairly steep, the variation of speed of these nodes is significant and results in oscillations in the mesh trajectories and other related quantities. Figure 6 shows that for this $\tau$, (MMPDE3) is most sensitive to these oscillations. Nevertheless, the mesh trajectory produced by (MMPDE3) follows the wave front (see Fig. 7(b)). The deviation from the equidistribution mesh is also related to these oscillations, and for the smaller value $10^{-5}$ for $\tau$, the oscillations are reduced. The resulting $E(t)$ for the MMPDEs is shown in Fig. 8, and the mesh trajectory for (MMPDE3) is plotted in Fig. 7(c). A remark is in order regarding the performance of (MMPDE7). Using 


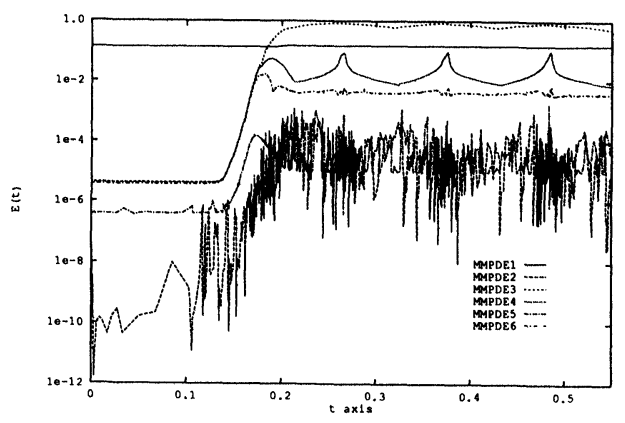

FIG. 8. Example 4.2: The function $E(t)$ obtained with the initial mesh UM and $\tau=10^{-5}$ is plotted for (MMPDEs 1-6).

TABLE 3

Example 4.2.

\begin{tabular}{|c|c|cccccc|}
\hline$\tau$ & MMPDE & 1 & 2 & 3 & 4 & 5 & 6 \\
\hline \multirow{2}{*}{$10^{-3}$} & nst & 809 & 894 & 1349 & 1075 & 1353 & 959 \\
\cline { 2 - 8 } & jac & 143 & 150 & 240 & 195 & 282 & 167 \\
\hline \multirow{2}{*}{$10^{-5}$} & nst & 809 & 1333 & 1369 & 1929 & 1463 & 1367 \\
\cline { 2 - 8 } & jac & 143 & 311 & 334 & 591 & 328 & 325 \\
\hline
\end{tabular}

several values for $\tau$ between 0.1 to $10^{-5}$, the ODE solver LSODI fails with an error message that the time step size is too small $\left(<10^{-8}\right)$. The reason is not completely clear, but it may be due to the oscillations mentioned above.

We find that for this problem that the meshes generated by (MMPDEs 1-6) are all quite stable. The results also show that with a small value for $\tau$ (MMPDEs 3-6) move the mesh to preserve equidistribution. For all of the computations performed, no mesh crossing occurs for this example. Summary information for nst and jac is listed in Table 3.

We conclude this section with a description of a method that uses the MMPDEs to generate an initial equidistribution mesh. To be specific, we only discuss it for the solution $u(x, 0)$ in (75) with $c(0)=10^{3}$. Since $u(x, 0)$ is quite steep at $x=0.4$, it is natural to employ a (pseudo-)time integration of the MMPDE to steady state with the resulting solution being the equidistribution mesh for $u(x, 0)$. For an illustration, we let

$$
c(t)= \begin{cases}t, & 0 \leq t \leq 10^{3}, \\ 10^{3}, & 10^{3}<t,\end{cases}
$$

discretize the MMPDE, and integrate the resulting ODE from $t=0$ to $10^{4}$. We use a relative error indicator

$$
R(t)=\max _{1 \leq i \leq n-1} \frac{1}{M_{i}(t)}\left|E_{i}(t)\right|
$$

here since $M(x, t)$ is very large. Computations are performed for (MMPDEs 1-6) with an initial uniform mesh and $\tau=10^{-3} . R(t)$ and $S(t)$ for (MMPDEs 1-6) are plotted in Fig. 9, and the mesh trajectories for (MMPDEs 1 and 2) are shown in Fig. 10. The corresponding values of nst and jac are listed in Table 4. 
(a)

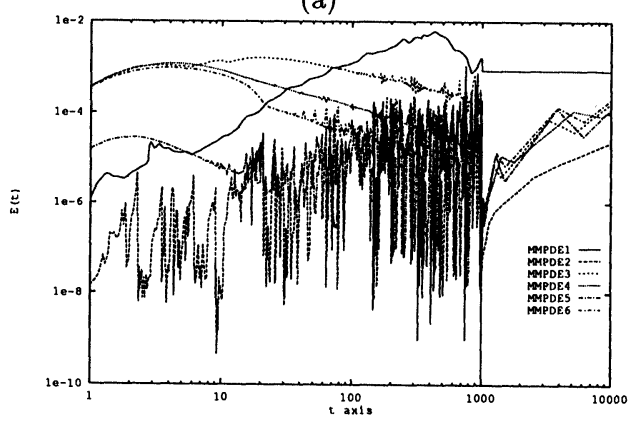

(b)

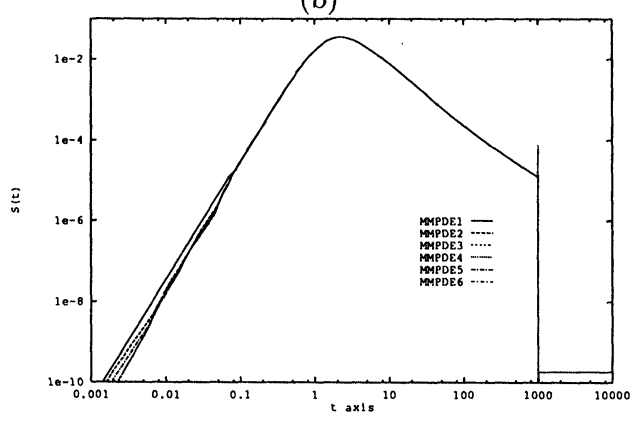

FIG. 9. Initial mesh generation: The functions $E(t)$ and $S(t)$ for (MMPDEs 1-6) with the initial mesh UM are plotted in (a) and (b), respectively. Here, $\tau=10^{-3}$ is used.

(a)

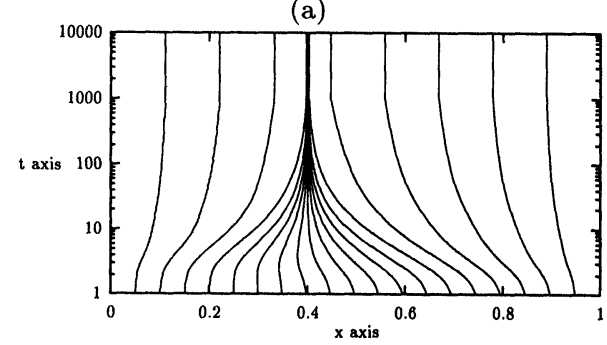

(b)

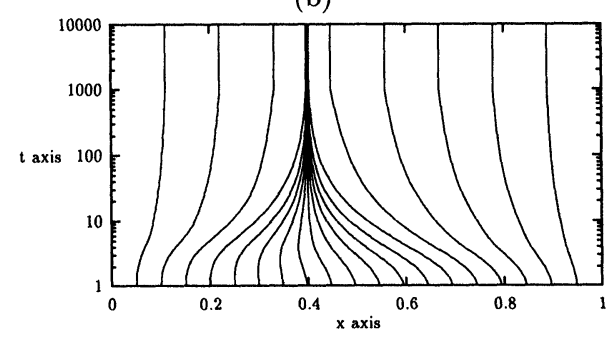

FIG. 10. Initial mesh generation: Mesh trajectories, which start with the initial uniform mesh, are generated (a) by (MMPDE1), (b) by (MMPDE2) with $\tau=10^{-3}$.

5. Conclusions and comments. Several MMPDEs related to the equidistribution principle have been derived and studied theoretically and numerically. It is seen that (MMPDE1), a commonly used approach, must be used with care. The deviation from the equidistribution mesh for (MMPDE1) depends strongly on the initial mesh, and mesh crossings can take place in some situations.

(MMPDE2) is shown to have several desirable properties. While it can easily be analyzed theoretically, a computational difficulty is that the function $\frac{\partial M}{\partial t}$ is needed (as it is for (MMPDE1)). Its simplified versions, (MMPDEs 3 and 4), not only have most of the properties of (MMPDE2), but also are much easier to use.

(MMPDEs 2-4 and 6) can all be easily shown to avoid mesh crossing in theory, and in our limited experience no mesh crossing has occurred when (MMPDEs 2-7) are discretized and the mesh computed numerically. The deviation from the equidistribution mesh associated with (MMPDEs 3-7) can be shown to be small for sufficiently small values of $\tau$. The correction term (21) in (MMPDEs 2-7) can be interpreted as a source of mesh movement, as a stablizing term, and as a mechanism to pull the mesh back toward equidistribution. We have developed, as a side benefit of the MMPDEs, a natural and practical way to generate an equidistribution mesh for the initial solution to a PDE.

The main purpose of these MMPDEs is of course to formulate simple, robust moving mesh methods that are solved along with an underlying PDE. In this paper we have only considered the MMPDE itself and seen that the discrete solutions generally seem to inherit the nice theoretical properties of the continuous solutions to 
TABLE 4

Initial mesh generation. $\tau=10^{-3}$ is used.

\begin{tabular}{|c|cccccc|}
\hline MMPDE & 1 & 2 & 3 & 4 & 5 & 6 \\
\hline nst & 303 & 589 & 558 & 581 & 541 & 572 \\
\hline jac & 49 & 129 & 126 & 134 & 111 & 136 \\
\hline
\end{tabular}

the MMPDEs. A moving finite difference method based on (MMPDEs 3-6) has been designed, and preliminary results for it, which will appear elsewhere, are promising. Moreover, extensions to higher space dimensions are currently underway.

\section{REFERENCES}

[1] S. Adjerid AND J. E. FlaherTy, A moving finite element method with error estimation and refinement for one-dimensional time dependent partial differential equations, SIAM J. Numer. Anal., 23 (1986), pp. 778-795.

[2] — A moving-mesh finite element method with local refinement for parabolic partial differential equations, Comput. Meth. Appl. Mech. Engrg., 55 (1986), pp. 3-26.

[3] D. A. Anderson, Adaptive mesh schemes based on grid speeds, AIAA Paper, 83-1931 (1983), p. 311 .

[4] - Application of adaptive grids to transient problems, in Adaptive Computational Methods for Partial Differential Equations, I. Babuška, J. Chandra, and J. E. Flaherty, eds., Society for Industrial and Applied Mathematics, Philadelphia, PA, 1983, pp. 208-223.

[5] C. DE BooR, Good approximation by splines with variable knots II, in Springer Lecture Notes Series 363, Springer-Verlag, Berlin, 1973.

[6] J. M. Coyle, J. E. Flaherty, AND R. Ludwig, On the stability of mesh equidistribution strategies for time-dependent partial differential equations, J. Comput. Phys., 62 (1986), pp. 26-39.

[7] E. A. Dorfi And L. O'C. DruRy, Simple adaptive grids for 1-D initial value problems, J. Comput. Phys., 69 (1987), pp. 175-195.

[8] J. E. Flaherty, J. M. Coyle, R. Ludwig, and S. F. Davis, Adaptive finite element methods for parabolic partial differential equations, in Adaptive Computational Methods for Partial Differential Equations, I. Babuška, J.Chandra, and J.E. Flaherty, eds., Society for Industrial and Applied Mathematics, Philadelphia, PA, 1983, pp. 144-164.

[9] R. M. Furzeland, J. G. Verwer, And P. A. Zegeling, A numerical study of three moving grid methods for one-dimensional partial differential equations which are based on the method of lines, J. Comput. Phys., 89 (1990), pp. 349-388.

[10] J. B. GreenberG, A new self-adaptive grid method, AIAA J., 23 (1985), pp. 317-320.

[11] D. F. HAWken, J. J. GotTliEb, AND J. S. HANSEN, Review of some adaptive node-movement techniques in finite element and finite difference solutions of PDEs, J. Comput. Phys., 95 (1991), pp. 254-302.

[12] B. M. Herbst, S. W. Schoombie, And A. R. Mrtchell, Equidistributing principles in moving finite element methods, J. Comput. Appl. Math., 9 (1983), pp. 377-389.

[13] A. C. HINDMARSH, LSODE and LSODI, two new initial value ordinary differential equation solvers, ACM SIGNUM Newsletter, 15 (1980), pp. 10-11.

[14] R. G. Hindman AND J. Spencer, A new approach to truly adaptive grid generation, AIAA Paper 83-0450, 1983, p. 1.

[15] W. HUANG AND D. M. Sloan, A simple adaptive grid method in two dimensions, SIAM J. Sci. Comput., 15 (1994), to appear.

[16] J. M. Hyman AND B. LARRouturou, Dynamic rezone methods for partial differential equations in one space dimension, Los Alamos, Rep. LA-UR-86-1678, 1986.

[17] N. K. MADSEN, MOLAG: A method of lines adaptive grid interface for nonlinear partial differential equations, in PDE Software: Modules, Interfaces and Systems, B. Engquist and T. Smedsaas, eds., North Holland, Amsterdam, 1984.

[18] K. Miller, Moving finite elements II, SIAM J. Numer. Anal., 18 (1981), pp. 1033-1057.

[19] K. Miller AND R. N. Miller, Moving finite elements I, SIAM J. Numer. Anal., 18 (1981), pp. 1019-1032. 
[20] Y. REN, Theory and computation of moving mesh methods for solving time-dependent partial differential equations, Ph.D. thesis, Department of Mathematics and Statistics, Simon Fraser University, Burnaby, Canada, 1992.

[21] Y. REN AND R. D. RuSSELL, Moving mesh techniques based upon equidistribution, and their stability, SIAM J. Sci. Statist. Comput., 13 (1992), pp. 1265-1286.

[22] A. B. WhITE, JR., On selection of equidistributing meshes for two-point boundary-value problems, SIAM J. Numer. Anal., 16 (1979), pp. 472-502. 